\title{
Short-term effects of transcatheter aortic valve implantation on left atrial appendage function
}

\author{
Serkan Aslan ${ }^{1}$, Mehmet Gul ${ }^{1}$, Huseyin Altug Cakmak ${ }^{2}$, Derya Ozturk ${ }^{1}$, \\ Omer Celik ${ }^{1}$, Muhammet Hulusi Satilmisoglu ${ }^{1}$, Safa Gode ${ }^{3}$, \\ Omer Tasbulak ${ }^{1}$, Aydin Yildirim ${ }^{1}$, Ihsan Bakir ${ }^{3}$ \\ ${ }^{1}$ Department of Cardiology, Mehmet Akif Ersoy Thoracic and Cardiovascular Surgery \\ Training and Research Hospital, Istanbul, Turkey \\ ${ }^{2}$ Department of Cardiology, Rize Kackar Government Hospital, Rize, Turkey \\ ${ }^{3}$ Department of Cardiovascular Surgery, Mehmet Akif Ersoy Thoracic and Cardiovascular \\ Surgery Training and Research Hospital, Istanbul, Turkey
}

\begin{abstract}
Background: The beneficial effects of the transcatheter aortic valve implantation (TAVI) on echocardiographic parameters including left atrial (LA) and left ventricular (LV) functions were described by previous studies. The aim of this study was to analyze the effects of TAVI on left atrial appendage (LAA) function assessed by transthoracic and transesophageal echocardiography.
\end{abstract}

Methods: Fifty-five patients with severe symptomatic aortic stenosis were included in this prospective study. LAA early and late emptying velocities, LAA filling velocity, peak early diastolic $\left(E_{M}\right)$, late diastolic $\left(A_{M}\right)$, and systolic $\left(S_{M}\right)$ velocities were measured with pulsed wave Doppler and tissue Doppler imaging, and $E / E_{m}$ ratio was calculated before and $7.1 \pm 2.8$ days after TAVI. A subgroup analysis was performed in accordance with the left ventricular ejection fraction (LVEF) of the patients and the severity of their LV diastolic dysfunction.

Results: Although the post-procedure peaks and mean gradients of the patients decreased significantly, the LVEF increased significantly in those who had low LVEF before the procedure. The post-procedure $E / E_{m}$ ratio decreased significantly $(p<0.001)$. The post-procedural LAA mean filling velocity and $E_{M}$ velocity were significantly higher than the pre-procedural filling velocity ( $p<0.001, p=0.002$, respectively). In the subgroup analysis, the post-procedural LAA filling velocity, early and late LAA emptying velocities, in addition to the mean velocity of the $E_{M}$, $A_{M}$ and $S_{M}$ were significantly higher than before the procedure in patients with LVEF of $<50 \%$ and $E / E_{m}$ ratios of $>15$.

Conclusions: LAA function improved soon after the TAVI procedure, especially in patients with low LVEF and marked LV diastolic dysfunction. (Cardiol J 2015; 22, 5: 527-534)

Key words: transcatheter aortic valve implantation, left atrial appendage, echocardiography

Address for correspondence: Serkan Aslan, MD, Mehmet Akif Ersoy Thoracic and Cardiovascular Surgery Training and Research Hospital, Department of Cardiology, Istasyon Mah. Turgut Ozal Bulvari No: 11, 34303, Kucukcekmece, Istanbul, Turkey, tel: +905305149486, fax: +9021247194 94, e-mail: serkanaslan84@hotmail.com 


\section{Introduction}

Aortic stenosis (AS) leads to severe left ventricular (LV) hypertrophy and impaired relaxation of the LV, resulting in LV diastolic dysfunction [1]. In advanced-stage AS, the contractile functions of myocardial cells are reduced and LV systolic functions are impaired. The subsequent chronic pressure and volume overload into the LV adversely affect the functions of the left atrial (LA) and the LA appendage (LAA) [2, 3].

Transcatheter aortic valve implantation (TAVI) has emerged as an alternative method of surgical aortic valve replacement for symptomatic patients with severe AS and with very high or prohibitive operative risks [4]. Recent studies that used speckle-tracking echocardiography reported an improvement in some LA functions after TAVI in high-risk AS patients [5-7]. Although the beneficial effect of TAVI on clinical and echocardiographic hemodynamic parameters is well known, there are no data in the literature regarding the effect of this procedure on LAA functions. In this study, the functions of the LAA were compared before and approximately 1 week after the TAVI procedure pulsed wave (PW) Doppler and the tissue Doppler imaging (TDI) with transesophageal echocardiography (TEE) technique.

\section{Methods}

Study design and patient selection: This study enrolled 78 consecutive patients from January 2011 to December 2014 who had symptomatic aortic valve stenosis New York Heart Association (NYHA) functional class $\geq$ II and documented calcific aortic valve stenosis, with an initial aortic valve area (AVA) of $<1.0 \mathrm{~cm}^{2}$ (or an AVA index of $<0.6 \mathrm{~cm}^{2} / \mathrm{m}^{2}$ ) and a mean pressure gradient of $>40 \mathrm{~mm} \mathrm{Hg}$ or a jet velocity of $>4 \mathrm{~m} / \mathrm{s}$, as measured by transthoracic echocardiography (TTE). The patients were deemed high risk based on the Society of Thoracic Surgery (STS) score of $\geq 10 \%$, a logistic EuroSCORE of $\geq 20 \%$, or agreement by a multidisciplinary heart team that frailty and/or coexisting comorbidities would be associated with a high surgical risk $[8,9]$. The study's exclusion criteria were the following: a congenital unicuspid or bicuspid aortic valve, an acute myocardial infarction, a transient ischemic attack, or a stroke within previous 6 months. Additional exclusion criteria were any permanent neurological defects, severe renal insufficiency, a pre-existing prosthetic heart valve or a prosthetic ring in any position, more than moderate $(>3+)$ mitral regurgitation or aortic regurgitation, untreated clinically significant coronary artery disease and a documented LV ejection fraction (LVEF) below 30\%. In patients with a thrombus or severe spontaneous echo contrast $(>2+)$ in the LAA, which is a contraindication for the TAVI procedure, were excluded from this study. After the initial evaluation, 23 subjects were excluded from further analysis. Finally, 55 patients were included in the study. Patient demographics and medical history were obtained by the attending physicians. The Edwards Sapien XT transcatheter aortic valve system (Edwards Life Sciences, Irvine, CA, US) was used in all cases, with the patients under general anesthesia. The study complied with the Declaration of Helsinki, and the trial protocol was approved by the Institutional Ethics Committee of the hospital.

\section{Echocardiographic study}

All the patients underwent TTE and TEE (General Electric Vivid 7 GE Vingmend Ultrasound AS, Horten, Norway) before the procedure and again the first week after TAVI, according to the recommendations of the American Society of Echocardiography [10-11]. Two independent experienced physicians who were blinded to the patients' clinical characteristics performed all the echocardiographic measurements. The patients' blood pressure was measured prior to all of the procedures.

\section{TTE parameters}

LV systolic function was assessed by calculating the patients' LVEF with 4-chamber Simpson's method. LV diastolic performance was evaluated using PW Doppler and TDI. The LV diastolic inflow velocities were obtained from the apical 4-chamber view by placing the sample volume at the level of the mitral valve tip. Peak early diastolic flow (E) and peak atrial filling flow velocities (A) were measured. TDI of the velocities of longitudinal mitral annular motion were recorded at the septal and lateral mitral annular borders, the average of the results obtained. The early diastolic $\left(\mathrm{E}_{\mathrm{m}}\right)$, and late diastolic $\left(\mathrm{A}_{\mathrm{m}}\right)$ Doppler tissue velocities of the mitral annulus were measured, and the ratio of mitral inflow E velocity to tissue Doppler $\left(\mathrm{E} / \mathrm{E}_{\mathrm{m}}\right)$ for both septal and lateral walls was calculated, the average of the results was obtained.

\section{TEE parameters}

All the patients were instructed not to take water or food for at least $4 \mathrm{~h}$. The patients' oro- 


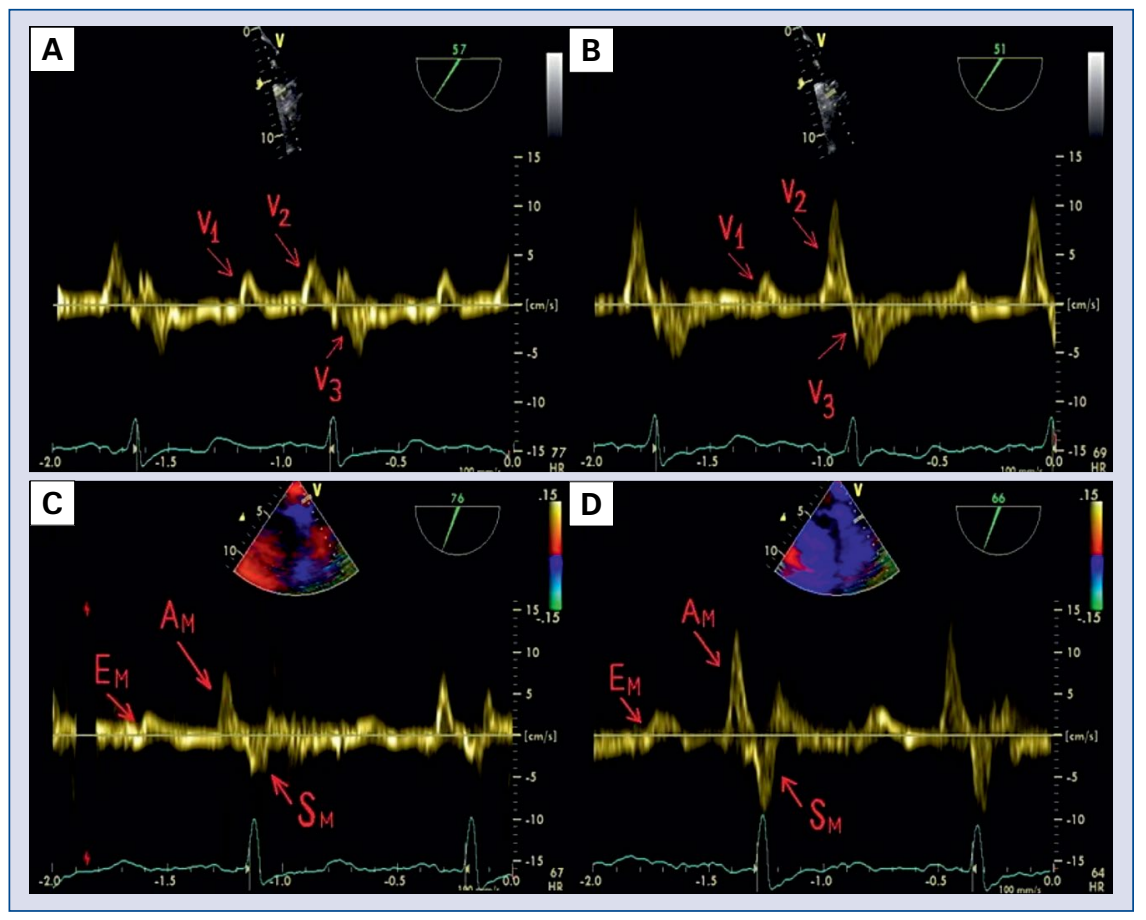

Figure 1. Effect of transcatheter aortic valve implantation on left atrial appendage Doppler velocities: before (A), after (B) and Doppler tissue velocities: before (C), after (D).

pharynx was anesthetized with a topical lidocaine $10 \%$ spray. Sedation with midazolam was used if needed. TEE was performed with a $5 \mathrm{MHz}$ multiplane transesophageal probe. The LAA was visualized in the mid-esophageal 2-chamber view by rotation of the imaging sector from $50^{\circ}$ to $90^{\circ}$. The LAA emptying and filling flow velocities were also recorded by PW Doppler, with a $2 \times 2 \mathrm{~mm}$ sampling volume placed at the one-third proximal position of the LAA cavity. During the assessment of LAA flow, 2 blood flow velocities were evaluated. An early diastolic outflow wave observed before the electrocardiographic $\mathrm{P}$ wave was termed $\mathrm{V}_{1}$. Biphasic waves following $V_{1}$ subsequent to the $P$ wave were termed $V_{2}$ (late emptying flow velocity) and $V_{3}$ (filling flow velocity) (Fig. 1). The peak flow velocities of the $V_{1}, V_{2}$, and $V_{3}$ waves were measured.

\section{LAA TDI velocities}

In the TDI analysis, the spectral mode of the PW Doppler sample volume of the LAA lateral wall was placed midway between the LAA tip and outlet. In sinus rhythm, 3 distinct velocities were recorded. The first positive wave after the electrocardiographic $\mathrm{P}$ wave was termed $\mathrm{A}_{\mathrm{M}}$, followed by a negative wave $\left(S_{M}\right)$, which coincided with $L V$ contraction, and then a positive wave $\left(\mathrm{E}_{\mathrm{M}}\right)$ during
LV relaxation. A representative illustration of the LAA TDI velocities of a patient pre- and post-TAVI is shown in Figure 1.

\section{Statistical analysis}

Continuous variables are expressed as mean \pm standard deviation. Categorical variables were expressed as frequencies and/or percentages. The Kolmogorov-Smirnov test was used to evaluate whether the continuous variables were normally distributed. The "paired samples test" was used to compare the before and after TAVI procedure results. A p value of less than 0.05 was considered to indicate statistical significance. Statistical analyses were carried out using the Number Cruncher Statistical System 2007 Statistical Software (Utah, USA).

\section{Results}

The baseline characteristics of the study patients are presented in Table 1 . The mean age was $77.3 \pm 6.9$ years, and $36(65 \%)$ patients were female. The mean logistic EuroSCORE and STS score were $27.5 \pm 14.2$ and $12.3 \pm 6.6$, respectively. Before the TAVI procedure, the diameter of the LA was $43.7 \pm 6.0 \mathrm{~mm}$ and that of the aortic valve effective orifice area was $0.76 \pm 0.17 \mathrm{~cm}^{2}$. When systolic blood pressures were compared in 
Table 1. Baseline patient characteristics ( $n=55)$.

\begin{tabular}{|c|c|}
\hline Variable & Value \\
\hline Age [years] & $77.3 \pm 6.9$ \\
\hline Female & $36(65 \%)$ \\
\hline Logistic EuroSCORE [\%] & $27.5 \pm 14.2$ \\
\hline STS score [\%] & $12.3 \pm 6.6$ \\
\hline Body mass index $\left[\mathrm{kg} / \mathrm{m}^{2}\right]$ & $28.0 \pm 4.5$ \\
\hline Hypertension & $39(70 \%)$ \\
\hline Diabetes & $26(47 \%)$ \\
\hline Coronary artery disease & $33(60 \%)$ \\
\hline Cerebrovascular event & $4(7 \%)$ \\
\hline Smoker & $18(32 \%)$ \\
\hline Creatinine $[\mathrm{mg} / \mathrm{dL}]$ & $1.0 \pm 0.7$ \\
\hline LDL-C [mg/dL] & $111 \pm 35$ \\
\hline $\mathrm{WBC}\left[\mathrm{k} / \mathrm{mm}^{3}\right]$ & $7.0 \pm 1.7$ \\
\hline HGB [g/dL] & $11.8 \pm 1.6$ \\
\hline LVEF $<50 \%$ & $21(39 \%)$ \\
\hline $\operatorname{LVDD}\left(E / E_{m}>15\right)$ & $22(40 \%)$ \\
\hline Effective orifice area $\left[\mathrm{cm}^{2}\right]$ & $0.76 \pm 0.17$ \\
\hline LA diameter [mm] & $43.7 \pm 6.0$ \\
\hline $\begin{array}{l}\text { Time to echocardiography } \\
\text { after implantation [days] }\end{array}$ & $7.1 \pm 2.8$ \\
\hline \multicolumn{2}{|l|}{$\begin{array}{l}\text { Aortic valve prosthesis: } \\
\text { - Edwards SAPIEN XT: }\end{array}$} \\
\hline $23 \mathrm{~mm}$ & $18(32.7 \%)$ \\
\hline $26 \mathrm{~mm}$ & $23(41.8 \%)$ \\
\hline $29 \mathrm{~mm}$ & $14(25.5 \%)$ \\
\hline LA diameter [mm] & $43.7 \pm 6.0$ \\
\hline SBP $[\mathrm{mm} \mathrm{Hg}]:$ & $P=0.106$ \\
\hline Before & $125 \pm 12.3$ \\
\hline After & $132 \pm 13.1$ \\
\hline
\end{tabular}

Mean values (standard deviation) and \% (n) are reported for continuous and categorical variables; STS - Society of Thoracic Surgery score; LDL-C - low density lipoprotein cholesterol; WBC - white score; $\mathrm{LDL}-\mathrm{C}$ - low density lipoprotein cholesterol; WBC - whit
blood cell; HGB - hemoglobin; LVEF - eft ventricular ejection fraction; LVDD - left ventricular diastolic dysfunction; LA - left atrium; SBP - systolic blood pressure

terms of before vs. after TAVI procedure, no significant differences were found (before: $125 \pm 12.3$ mm Hg vs. after: $132 \pm 13.1 \mathrm{~mm} \mathrm{Hg}, \mathrm{p}=0.106$ ).

\section{TTE}

TTE 2-dimensional and Doppler parameters before and after the procedure are given in Table 2 . The mean and peak pressure gradients were significantly reduced after the TAVI procedure $(\mathrm{p}<0.001)$. Moreover, there was no difference between preand post-procedural LVEFs in the patients with $\operatorname{AS}(p=0.054)$. However, in the subgroup analysis, the post-procedural LVEF was higher in the low LVEF group $(\mathrm{p}=0.003)$. The post-procedural means of the $\mathrm{E} / \mathrm{E}_{\mathrm{m}}$ were also significantly lower compared to the pre-procedural means $(\mathrm{p}<0.001)$.

\section{TEE}

The comparison of the TEE PW Doppler and TDI parameters of pre- and post-procedural LAA functions in all the patients is reported in Table 2. Subgroup analyses were performed based on the LVEF (LVEF < 50\% and LVEF > 50\%) (Table 3) and diastolic dysfunction parameters $\left(\mathrm{E} / \mathrm{E}_{\mathrm{m}}>15\right.$ and $\mathrm{E} / \mathrm{E}_{\mathrm{m}}<15$ ) of the patients (Table 4 ). The post-procedural means of LAA filling velocities were significantly higher compared to the pre-procedural means in all study groups $(\mathrm{p}<0.001)$. When the results of the subgroup analyses of the low and preserved LVEF groups were compared, the post-procedural means of LAA filling velocities were significantly higher compared to the pre-procedural means in both the low and preserved LVEF groups $(\mathrm{p}=0.001$ and $\mathrm{p}=0.021$, respectively). In the low LVEF group, the post-procedural means of the LAA early and late emptying velocities were significantly higher compared to the pre-procedural means ( $p=0.022$ and $p=0.036$, respectively). However, the post-procedural means of the LAA early and late emptying velocities were not different from the pre-procedural means in the preserved LVEF group. In the TDI analysis, although the post-procedural velocities of the peak early diastolic $\left(\mathrm{E}_{\mathrm{M}}\right)$, peak late diastolic $\left(\mathrm{A}_{\mathrm{M}}\right)$, and peak systolic $\left(\mathrm{S}_{\mathrm{M}}\right)$ were increased, only the increase in the $\mathrm{E}_{\mathrm{M}}$ velocity was significantly higher $(\mathrm{p}=0.002)$. In the subgroup analysis, the post-procedural velocities of $E_{M}, A_{M}$, and $S_{M}$ were significantly increased in the low LVEF group (Table 3). Despite the increase in the post-procedural velocities of these parameters, there was no significant difference between the pre- and post-procedural velocities in the preserved LVEF group (Table 3). Moreover, the post-procedural means of LAA filling, early and late emptying velocities were significantly higher than the pre-procedural means in the patients with diastolic dysfunction $\left(\mathrm{E} / \mathrm{E}_{\mathrm{m}}>15\right)$. In the TDI analysis, the post-procedural velocities of $\mathrm{E}_{\mathrm{M}}, \mathrm{A}_{\mathrm{M}}$, and $\mathrm{S}_{\mathrm{M}}$ were significantly increased in the patients with diastolic dysfunction $\left(\mathrm{E} / \mathrm{E}_{\mathrm{m}}>15\right)$ (Table 4). However, there was no significant difference in the post-procedural LAA filling and emptying velocities or the LAA tissue Doppler velocities in the patients with preserved diastolic function $\left(\mathrm{E} / \mathrm{E}_{\mathrm{m}}<15\right)($ Table 4). 
Table 2. Comparison of TTE and TEE variables before and after TAVI.

\begin{tabular}{|c|c|c|c|}
\hline & Before & After & $\mathbf{P}$ \\
\hline \multicolumn{4}{|l|}{ TTE: 2D and Doppler parameters } \\
\hline \multicolumn{4}{|l|}{ LVEF [\%]: } \\
\hline All & $53.1 \pm 12.3$ & $54.3 \pm 10.2$ & 0.054 \\
\hline$<50 \%$ & $39.4 \pm 8.4$ & $43.1 \pm 7.2$ & 0.003 \\
\hline$>50 \%$ & $61.6 \pm 3.2$ & $61.2 \pm 3.6$ & 0.319 \\
\hline Peak aortic gradient [mm Hg] & $84.2 \pm 21.7$ & $18.8 \pm 9.6$ & $<0.001$ \\
\hline Mean aortic gradient [mm Hg] & $52.8 \pm 14.3$ & $10.3 \pm 5.0$ & $<0.001$ \\
\hline E velocity $[\mathrm{cm} / \mathrm{s}]$ & $95.5 \pm 34.3$ & $91.0 \pm 32.7$ & 0.230 \\
\hline $\mathrm{E}_{\mathrm{m}}$ velocity $[\mathrm{cm} / \mathrm{s}]$ & $7.1 \pm 2.1$ & $8.0 \pm 2.2$ & $<0.001$ \\
\hline$E / E_{m}$ & $13.7 \pm 4.6$ & $11.5 \pm 4.1$ & $<0.001$ \\
\hline \multicolumn{4}{|c|}{ TEE: Doppler and TDI parameters } \\
\hline LAA emptying velocity [cm/s] & $37.8 \pm 16.1$ & $39.5 \pm 16.1$ & 0.129 \\
\hline LAA filling velocity $[\mathrm{cm} / \mathrm{s}]$ & $38.8 \pm 10.6$ & $42.3 \pm 12.0$ & $<0.001$ \\
\hline $\mathrm{E}_{\mathrm{M}}[\mathrm{cm} / \mathrm{s}]$ & $7.8 \pm 3.8$ & $8.9 \pm 4.0$ & 0.002 \\
\hline $\mathrm{A}_{\mathrm{M}}[\mathrm{cm} / \mathrm{s}]$ & $14.4 \pm 6.1$ & $15.6 \pm 6.2$ & 0.065 \\
\hline $\mathrm{S}_{\mathrm{M}}[\mathrm{cm} / \mathrm{s}]$ & $13.1 \pm 6.0$ & $14.2 \pm 6.3$ & 0.091 \\
\hline
\end{tabular}

Mean values (standard deviation) are reported for continuous variables; TTE — transthoracic echocardiography; TEE - transesophageal echocardiography; TAVI — transcatheter aortic valve implantation; LAA — left atrial appendage; TDI — tissue Doppler imaging

Table 3. Comparison of LAA function before and after TAVI by TEE with LVEF.

\begin{tabular}{lccc}
\hline Parameter & Before & After & P \\
\hline LVEF > 50\% & & & 0.578 \\
LAA emptying velocity [cm/s] & $46.0 \pm 14.0$ & $46.8 \pm 15.8$ & $\mathbf{0 . 0 2 1}$ \\
LAA filling velocity [cm/s] & $43.3 \pm 9.9$ & $45.8 \pm 11.7$ & 0.182 \\
$E_{M}[\mathrm{~cm} / \mathrm{s}]$ & $9.4 \pm 3.5$ & $10.1 \pm 3.7$ & 0.533 \\
$A_{M}[\mathrm{~cm} / \mathrm{s}]$ & $16.9 \pm 5.4$ & $17.5 \pm 6.1$ & 0.865 \\
$\mathrm{~S}_{M}[\mathrm{~cm} / \mathrm{s}]$ & $16.0 \pm 5.7$ & $16.1 \pm 6.7$ & $\mathbf{0 . 0 3 6}$ \\
LVEF <50\% & & & $\mathbf{0 . 0 0 1}$ \\
LAA emptying velocity [cm/s] & $24.6 \pm 9.0$ & $27.6 \pm 7.2$ & $<\mathbf{0 . 0 0 1}$ \\
LAA filling velocity [cm/s] & $31.5 \pm 7.4$ & $36.6 \pm 10.3$ & $\mathbf{0 . 0 0 7}$ \\
$E_{M}[\mathrm{~cm} / \mathrm{s}]$ & $5.2 \pm 2.6$ & $7.2 \pm 3.7$ & $<0.001$ \\
$A_{M}[\mathrm{~cm} / \mathrm{s}]$ & $9.2 \pm 3.6$ & $11.8 \pm 4.6$ & $11.0 \pm 3.9$ \\
$\mathrm{~S}_{M}[\mathrm{~cm} / \mathrm{s}]$ & $8.6 \pm 2.9$ & & \\
\hline
\end{tabular}

Mean values (standard deviation) are reported for continuous variables. LAA — left atrial appendage; TEE — transesophageal echocardiography; TAVI - transcatheter aortic valve implantation; LVEF — left ventricular ejection fraction

\section{Discussion}

Previous studies described the beneficial effects of the TAVI procedure on clinical, hemodynamic, and echocardiographic parameters including LA and LV functions, in patients with severe AS [12-15]. However, there are no data in the literature regarding the effects of TAVI on the functions of the LAA. In this study, we used TTE and TEE to evaluate pre- and post-procedural $(7.1 \pm 2.8$ days $)$ LAA functions. We demonstrated, for the first time, an improvement in LAA functions detected by PW Doppler and TDI approximately 1 week after the TAVI procedure, especially in patients with marked diastolic dysfunction $\left(\mathrm{E} / \mathrm{E}_{\mathrm{m}}>15\right)$ and a low LVEF $(<50 \%)$.

In advanced-stage AS, volume overload, which is caused by a depressed LVEF, impairs LA and 
Table 4. Comparison of LAA function before and after TAVI by TEE with $E / E_{m}$.

\begin{tabular}{|c|c|c|c|}
\hline Parameter & Before & After & $\mathbf{P}$ \\
\hline \multicolumn{4}{|l|}{$E / E_{m}>15$} \\
\hline LAA emptying velocity $[\mathrm{cm} / \mathrm{s}]$ & $30.6 \pm 13.4$ & $36.1 \pm 16.7$ & 0.001 \\
\hline LAA filling velocity $[\mathrm{cm} / \mathrm{s}]$ & $35.5 \pm 9.8$ & $41.7 \pm 11.4$ & $<0.001$ \\
\hline $\mathrm{E}_{\mathrm{M}}[\mathrm{cm} / \mathrm{s}]$ & $6.6 \pm 3.0$ & $9.0 \pm 3.7$ & $<0.001$ \\
\hline$A_{M}[\mathrm{~cm} / \mathrm{s}]$ & $11.8 \pm 4.9$ & $15.2 \pm 6.0$ & $<0.001$ \\
\hline $\mathrm{S}_{\mathrm{M}}[\mathrm{cm} / \mathrm{s}]$ & $11.3 \pm 5.3$ & $14.3 \pm 6.1$ & $<0.001$ \\
\hline \multicolumn{4}{|l|}{$E / E_{m}<15$} \\
\hline LAA emptying velocity $[\mathrm{cm} / \mathrm{s}]$ & $42.6 \pm 16.1$ & $41.8 \pm 15.6$ & 0.552 \\
\hline LAA filling velocity $[\mathrm{cm} / \mathrm{s}]$ & $41.0 \pm 10.7$ & $42.7 \pm 12.5$ & 0.091 \\
\hline $\mathrm{E}_{\mathrm{M}}[\mathrm{cm} / \mathrm{s}]$ & $8.6 \pm 4.0$ & $8.9 \pm 4.2$ & 0.490 \\
\hline $\mathrm{A}_{\mathrm{M}}[\mathrm{cm} / \mathrm{s}]$ & $15.9 \pm 6.3$ & $15.8 \pm 6.4$ & 0.829 \\
\hline $\mathrm{S}_{\mathrm{M}}[\mathrm{cm} / \mathrm{s}]$ & $14.4 \pm 6.2$ & $14.0 \pm 6.5$ & 0.683 \\
\hline
\end{tabular}

Mean values (standard deviation) are reported for continuous variables; LAA — left atrial appendage; TEE — transesophageal echocardiography; TAVI — transcatheter aortic valve implantation

LAA functions. This impairment leads to a decrease in the LAA velocities, the LAA Doppler tissue velocities, and the mechanical functions between the LA-LAA $[2,16,17]$. LV function is an important predictor of LAA velocity. A previous study reported a relationship between a low LVEF and LAA dysfunction. Moreover, reduced LAA velocities in non-valvular atrial fibrillation and LV systolic dysfunction were presented in recent studies [18-20].

Transcatheter valve implantation offers a new treatment modality for patients who have multiple comorbidities that make conventional surgery a high risk [21]. Recent studies reported an improvement in LA functions after the TAVI procedure in high-risk patients with systolic and diastolic dysfunction [5-7]. In the present study, the amelioration of LAA functions soon after the TAVI procedure may have been due to the marked and beneficial effects of the procedure on hemodynamic and echocardiographic parameters.

D'Andrea et al. [5] have recently used speckle-tracking echocardiography to demonstrate an improvement in the LA longitudinal function of patients with severe AS 6 months after they had undergone the TAVI procedure. Spethmann et al. [7] demonstrated that a global and regional LV and LA mechanics improved 12 months after TAVI. LA function was evaluated with speckle-tracking echocardiography in previous TAVI studies [5-7]. To the best of our knowledge, this is the first study to assess LAA functions after TAVI procedure in patients with severe AS. In this study, LAA filling velocities significantly increased in the post-procedural period. Although LAA emptying velocities were elevated after the TAVI procedure in this population, the increase was significant only in the low LVEF group. Spethmann et al. [6] demonstrated an improvement only in reservoir and conduit functions in the early period after the TAVI procedure. However, their study contained no information on the relationship between reservoir and conduit functions and LVEF. In this study, a significant increase in the post-procedural LAA tissue Doppler velocities in the low LVEF group was detected.

Different studies demonstrated depressed LA or LAA functions due to LV mass, LV outflow tract gradients, and LV diastolic dysfunction [22-24]. Moreover, the functions of LA booster-pump were found to be related to symptoms of severe AS in patients with preserved LVEF [22]. Although the velocities of the $\mathrm{E} / \mathrm{E}_{\mathrm{m}}$ and $\mathrm{E}_{\mathrm{m}}$ have a close relationship with systolic function, recent studies reported that this parameter reflected the functions of the LAA, independent of the LVEF [23-25].

In conclusion, the LA functions as a conduit of LV in early diastole and diastasis phases. Hence, it is affected directly by end-diastolic pressure and diastolic functions of the LV. The LA is also affected directly by compliance and pressure of the $L V$ because it functions as a pump of the $L V$ at the end of diastole [25-27]. In our study, the $\mathrm{E} / \mathrm{E}_{\mathrm{m}}$ was decreased in the post-procedural period. In the subgroup analysis, the post-procedural LAA filling velocity, LAA emptying velocity, and mean 
velocities of the $E_{M}, A_{M}$, and $S_{M}$ were significantly higher than those during the pre-procedural period in patients with an $\mathrm{E} / \mathrm{E}_{\mathrm{m}}$ of $>15$. However, this study has not revealed any difference in the post-procedural LAA functions in patients with an $\mathrm{E} / \mathrm{E}_{\mathrm{m}}$ of $<15$.

As a result of these data, the early increase in the functions of LAA may be due to improved post-procedural hemodynamic parameters, including elevated LVEFs and improved diastolic function, especially in patients with marked systolic and diastolic dysfunction. This improvement may be expected in long-term follow-up of patients with preserved LVEFs and diastolic function, because LA and LV remodeling takes much time.

\section{Limitations of the study}

This was a single-center study with a relatively small sample size, both of which limit the power of our research findings. Lack of follow-up of the patients in terms of short- and long-term cardiovascular events and link to the patients outcomes were the most serious weaknesses of our manuscript. Moreover, the lack of long-term follow-up of the echocardiographic parameters was another limitation of the present study. As atrial fibrillation is a common arrhythmia in patients with valvular heart disease, this was not an exclusion criterion. In our study, only 6 patients had chronic atrial fibrillation, and therefore $\mathrm{A}_{\mathrm{LAA}}$ was not evaluated in these patients.

\section{Conclusions}

In this study, we demonstrated that successful TAVI improved the echocardiographic hemodynamic status and increased the LAA Doppler and TDI velocities, which lead to improved regional LAA function in patients with a depressed LVEF, and increased LV diastolic dysfunction. Thus, the relief of AS by TAVI may not only confer hemodynamic benefits resulting in symptomatic improvement, but also have a favorable influence on LA and LAA function.

\section{Conflicts of interest: None declared}

\section{References}

1. Tziomalos K, Athyros VG, Karagiannis A, Mikhailidis DP. Established and emerging vascular risk factors and the development of aortic stenosis: an opportunity for prevention? Expert Opin Ther Targets, 2008; 12: 809-820. doi: 10.1517/14728222.12.7.809.
2. Zipes DP, Libby P, Bonow RO. Valvular Heart Diseases. In: Braunwald E ed. Braunwald's heart disease: A textbook of cardiovascular medicine. 7th Ed. W.B. Saunders Company, Philadelphia 2005: 1582-1592.

3. Kurt M, Wang J, Torre-Amione G, Nagueh SF. Left atrial function in diastolic heart failure. Circ Cardiovasc Imaging, 2009; 2: 10-15. doi: 10.1161/CIRCIMAGING.108.813071.

4. Vahanian A, Alfieri O, Andreotti F et al. Guidelines on the management of valvular heart disease. Joint Task Force on the Management of Valvular Heart Disease of the European Society of Cardiology (ESC); European Association for Cardio-Thoracic Surgery (EACTS). Eur Heart J, 2012; 33: 2451-2496. doi: 10.1093/eurheartj/ehs109.

5. D'Andrea A, Padalino R, Cocchia R et al. Effects of transcatheter aortic valve implantation on left ventricular and left atrial morphology and function. Echocardiography, 2015; 32: 928-936. doi: 10.1111/echo.12808.

6. Spethmann S, Dreger H, Baldenhofer G et al. Short-term effects of transcatheter aortic valve implantation on left atrial mechanics and left ventricular diastolic function. J Am Soc Echocardiogr, 2013; 26: 64-71.e2. doi: 10.1016/j.echo.2012.10.002.

7. Spethmann S, Baldenhofer G, Dreger H et al. Recovery of left ventricular and left atrial mechanics in various entities of aortic stenosis 12 months after TAVI. Eur Heart J Cardiovasc Imaging, 2014; 15: 389-398. doi: 10.1093/ehjci/jet166.

8. O'Brien SM, Shahian DM, Filardo G et al. The Society of Thoracic Surgeons 2008 cardiac surgery risk models: Part 2. Isolated valve surgery. Ann Thorac Surg, 2009; 88: S23-42. doi: 10.1016/j. athoracsur.2009.05.056.

9. Roques F, Michel P, Goldstone AR, Nashef SA. The logistic EuroSCORE. Eur Heart J, 2003; 24: 881-882.

10. Zamorano JL, Badano LP, Bruce $\mathrm{C}$ et al. EAE/ASE recommendations for the use of echocardiography in new transcatheter interventions for valvular heart disease. J Am Soc Echocardiogr, 2011; 24: 937-965. doi: 10.1016/j.echo.2011.07.003.

11. Hahn RT, Abraham T, Adams MS et al. Guidelines for performing a comprehensive transesophageal echocardiographic examination: recommendations from the American Society of Echocardiography and the Society of Cardiovascular Anesthesiologists. J Am Soc Echocardiogr, 2013; 26: 921-964. doi: 10.1016/j. echo.2013.07.009.

12. M. Gül, A. Yıldırım, N. Uslu et al. The outcomes of transcatheter aortic valve implantation with Edwards SAPIEN or CoreValve devices: Single-center experience in Turkey. Turkish J Thorac Cardiovasc Surg, 2012; 434-441. doi: 10.5606/tgkdc. dergisi.2012.087.

13. Gul M, Uyarel H, Akgul O et al. Hematologic and clinical parameters after transcatheter aortic valve implantation (TAVI) in patients with severe aortic stenosis. Clin Appl Thromb Hemost, 2014; 20: 304-310. doi: 10.1177/1076029612462762.

14. El-Mawardy M, Wübken-Kleinfeld N, Schwarz B et al. Transcatheter aortic valve implantation in patients with severely reduced left ventricular systolic function: a single-center experience. Clin Res Cardiol, 2014; 103: 621-630. doi: 10.1007/s00392-0140691-5.

15. Ayhan H, Kasapkara HA, Durmaz T et al. Impact of transcatheter aortic valve implantation in patients with reduced ejection fraction. Cardiol J, 2015; 22: 108-114. doi: 10.5603/CJ.a2014. 0040 . 
16. Aronow WS. Valvular aortic stenosis in the elderly. Cardiol Rev, 2007; 15: 217-225.

17. Elahi MM, Chuang A, Ewing MJ et al. One problem two issues! Left ventricular systolic and diastolic dysfunction in aortic stenosis. Ann Transl Med, 2014; 2: 10. doi: 10.3978/j.issn.23055839.2013.06.05.

18. Handke M, Harloff A, Hetzel A, Olschewski M, Bode C, Geibel A. Predictors of left atrial spontaneous echocardiographic contrast or thrombus formation in stroke patients with sinus rhythm and reduced left ventricular function. Am J Cardiol, 2005; 96: 1342-1344. doi: 10.1016/j.amjcard.2005.06.085

19. Cemri M, Timurkaynak T, Özdemir M et al. Effects of left ventricular systolic dysfunction on left atrial appendage and left atrial functions in patients with chronic nonvalvular atrial fibrillation. Acta Cardiol, 2002; 57: 101-105. doi: 10.5152/akd.2014.4833.

20. Tabata T, Oki T, Fukuda N, et al. Influence of left atrial pressure on left atrial appendage flow velocity patterns in patients in sinus rhythm. J Am Soc Echocardiography, 1996; 9: 857-864. doi: 10.1016/S0894-7317(96)90478-2

21. Leon MB, Smith CR, Mack M et al. Transcatheter aortic-valve implantation for aortic stenosis in patients who cannot undergo surgery. N Engl J Med, 2010; 363: 1597-1607. doi: 10.1056/ NEJMoa1008232.

22. Imanishi J, Tanaka H, Sawa $\mathrm{T}$ et al. Association of left atrial booster-pump function with heart failure symptoms in patients with severe aortic stenosis and preserved left ventricular ejection fraction. Echocardiography, 2015; 32: 758-767. doi: 10.1111/ echo.12733.

23. Doukky R, Garcia-Sayan E, Gage $\mathrm{H}$ et al. The value of diastolic function parameters in the prediction of left atrial appendage thrombus in patients with nonvalvular atrial fibrillation. Cardiovasc Ultrasound, 2014; 12: 10. doi: 10.1186/1476-712012-10.

24. Lee JS, Shim CY, Wi J et al. Left ventricular diastolic function is closely associated with mechanical function of the left atrium in patients with paroxysmal atrial fibrillation. Circ J, 2013; 77: 697-704. doi: 10.1253/circj.CJ-12-1009.

25. Hu YF, Hsu TL, Yu WC, et al. The impact of diastolic dysfunction on the atrial substrate properties and outcome of catheter ablation in patients with paroxysmal atrial fibrillation. Circ J, 2010; 74: 2074-2078. doi: 10.1253/circj.CJ-10-0175.

26. Jais P, Peng JT, Shah DC, et al. Left ventricular diastolic dysfunction in patients with so-called lone atrial fibrillation. J Cardiovasc Electrophysiol, 2000; 11: 623-625. doi: 10.1111/j.15408167.2000.tb00023.x.

27. Agmon Y, Khandheria BK, Meissner I et al. Are left atrial appendage flow velocities adequate surrogates of global left atrial function? A population-based transthoracic and transesophageal echocardiographic study. J Am Soc Echocardiogr, 2002; 15: 433-440. doi: 10.1067/mje.2002.116826. 